\title{
EVOLUTION OF A MOBILE ROBOT'S NEUROCONTROLLER ON THE GRASPING TASK Is Genetic also Generic?
}

\author{
Philippe Lucidarme \\ Lisa, University of Angers, France \\ philippe.lucidarme@univ-angers.fr
}

\begin{abstract}
Keywords: Evolutionary algorithm, mobile robot, artificial neural networks controller, grasping task.
Abstract: $\quad$ This paper presents a survey on the generic evolution of mobile robot' neurocontrollers with a particular focus on the capacity to adapt these controllers in several environments. Several experiments on the example of the grasping task (autonomous vacuum cleaner for example) are performed and the results show that the produced neurocontroller is dedicated to the trained conditions and cannot be considered as generic. The last part of the paper discusses of the necessary changes in the fitness function in order to produce generic neurocontrollers.
\end{abstract}

\section{INTRODUCTION}

In the past years, biology has been an inspiration for computer science researches. Genetics algorithms and artificial neural networks are probably the best illustration. Evolutionary algorithms are today used to optimize, classify or control in a large number of problems. The efficiency of the methods has been experimentally and sometime theoretically proven on given problems (Jansen, 2002), (Bäck, 1996) and (Floreano, 1994). In the particular field of robotics, especially in the case of real robots, proving the efficiency of the methods is very hard due to the complexity of the interactions between the environment and the robot. In spite of this, evolutionary algorithms are commonly used to optimize the parameters of robot's controllers.

The first experiment in the field of robotics has been performed in 1994 by Dario Floreano and Francesco Mondada (Floreano, 1994). Based on the used of a genetic algorithm, the aim of this experiment was to optimize the parameters of an artificial neural controller in order to generate an obstacle avoidance behavior. The experiment was performed on a real Kephera robot (Mondada 1993) and required 100 generations of 80 individuals. Each generation lasts 39 minutes. The results were remarkable, after 50 generations ( 32 hours) the robot already performed a behavior close to the optimal. Note that the Khepera robot is not a symmetrical robot: front face has 6 proximity sensors versus 2 for the rear face. During the evolution, the robot naturally selected the front face as the best direction. Few years later, the same team extended the experiment on a bigger robot with a different proximity sensors disposition (Floreano 1998). They continued the evolution on a Koala robot (described in section 4.4 of (Nolfi 2000)). In approximately thirty generations the best individuals reported fitness values similar to the experiment previously described with the Khepera robot (Floreano, 1994).

The first experiment proved the possibility of using evolutionary algorithm in order to learn basic behaviors on real robots. The second experiment proved that the previous results are platform independent, and this result can even be extended: from the neurocontroller point of view, inputs are the proximity sensors, and outputs are motor's commands. It means that the geometry and the kinematics of the robot are external to the controller. Then, the results of this second experiment can be extended to the environment, as the authors explained in (Nolfi 2000): "From the point of view of the neurocontroller, changing the sensory motor characteristics of the robot is just another way of modifying the environment".

These results have been exploited on several experiments like motion planning (Ahuactzin, 1992) or humanoid walking (. Yamasaki 2002) with a recurrent argument: the adaptive capacity of the evolutionary algorithms. According to the previous explanations, this capacity cannot be contested, but what about the neurocontroller? In fact, the 
neurocontroller is not generic; it has been optimized for the environment where the experiment has been performed. It means that if the robot needs to evolve in a different environment the evolutionary process needs to be restarted like for the Koala robot. In practice, and especially on real robots, the evolutionary algorithms need to be stopped to avoid performing dump behaviors due to unfortunate crossovers. The study presented in this paper evaluates the faculty of a neurocontroller to be adapted in several environments.

Second section of the paper describes the context of the experiment, based on a grasping task (Arkin, 1992). Introduced by R.C. Arkin in the 90's, this task consists in exploring a given environment (for example for mowing or painting the floor).

From section three, the following of the survey is based on a methodology inspired from biology: evolutionary algorithms are used to generate eight neurocontrollers in eight different environments. These neurocontrollers are stored as standard behavior and are compared in the seven other environments to evaluate there performances in different contexts.

The fourth part of the paper introduces a new experiment where the robot is trained in the eight environments: the fitness function is the average performance. Results are analyzed and compared with the standards previously defined.

The last part of the paper introduces a new fitness function based on the performance in the worst environment. A general conclusion ends the paper.

\section{EVOLUTIONARY ALGORITHMS ON THE GRASPING TASK}

\subsection{Grasping Task}

As explained in the introduction, the grasping task has been introduced in a paper on multi-agents systems in 1992 by R.C. Arkin. This task has been choosen because the duration of the evaluation of one individual can be bounded and also because the fitness function is easy to evaluate in simulation (explored area divided by total surface). Note that another kind of tasks would have been used like obstacles avoidance or target tracking.

Due to the duration of the experiments, all the presented results are obtained by simulation. Note also that our purpose in not to obtain an efficient neurocontroller on real robots but to compare the results in several contexts. Real experiments suffer from noise on sensors, wheels slipping or battery discharge that make the comparison sometime difficult. In spite of this, the simulator computes the model of the real robot Type 1 (described in the next section) and the environments are scaled around this robot. The simulator is designed with a library of eight environments. Each environment has a squared shape (length of the side: 3 meters). The disposal of walls has been chosen so as to do three kinds of environments: with large spaces (Figure 1.a,b,c and f), with narrow corridors (Figure 1.d and g.) and mixed (Figure 1.e and $\mathrm{h}$ ).

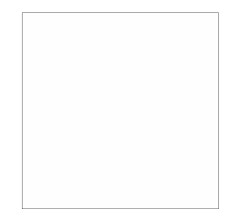

a.

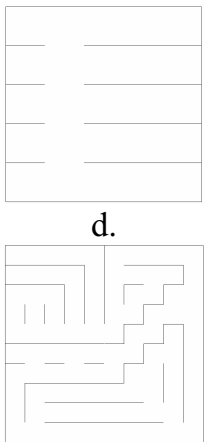

g.

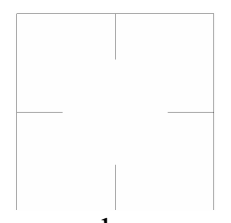

b.

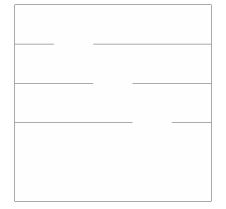

e.

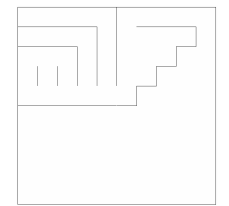

h.

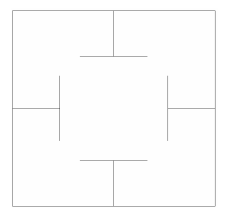

c.

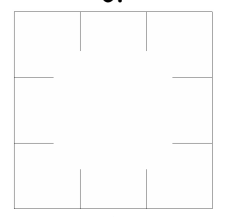

f.
Figure 1: The eight environments used to compare the learned behaviors.

\subsection{The Mobile Robot Type 1}

The kinematics model of the robot and sensor's disposal are similar to the robot Type 1 described in (Lucidarme, 2006). It has a $10 \mathrm{~cm}$-height and $13 \mathrm{~cm}$ diameter cylindrical shape (figure 5). Two wheels actuate it. Two small passive ball-in-socket units ensure the stability in place of usual castor-wheels. DC motors equipped with incremental encoders (352 pulses per wheel's revolution) control the wheels. The encoders may be used for both speed control and robot localization by odometry. The robot is surrounded with 16 infrared emitters and 8 receivers (shown on figure 2 and 3 ). The sensors use a carrier frequency of $40 \mathrm{kHz}$ for a good noise rejection. An embedded PC (80486 DX with $66 \mathrm{MHz}$ clock) manages the robot.

Figure 3. shows the model used in the simulator, especially the sensor's positions. The kinematics model used in the simulator is described by equation 1. This robot has been chosen because in the case where real experiments would have been necessary, 
Type 1 has many of the characteristics required by the evolutionary approach: fully embedded computation power ( $\mathrm{x} 86$ processor), up to two hours of autonomy (Li-ion batteries) and large memory capacity (compact-flash).

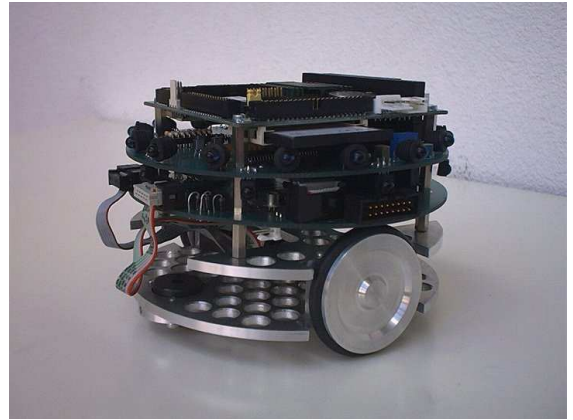

Figure 2: Picture of the mobile robot Type 1 equipped with infrared proximity sensors and the embedded PC104 visible on the top.

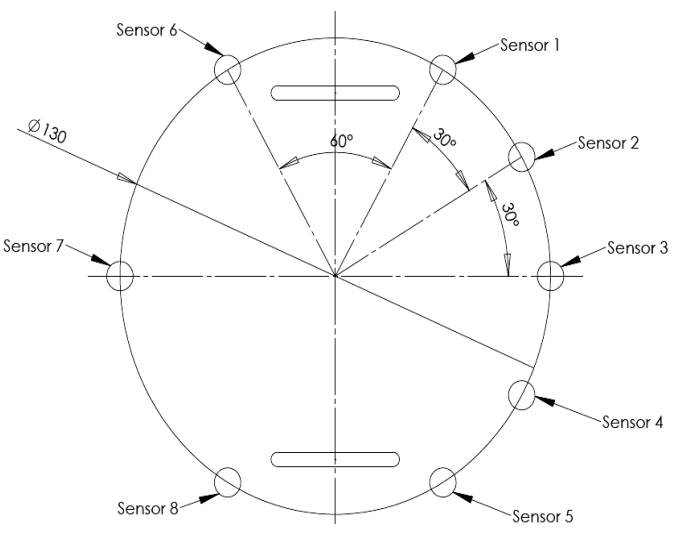

Figure 3: Description of the model used in the simulator and position of the sensors (Dimensions are in $\mathrm{mm}$ ).

$$
\left[\begin{array}{l}
\frac{d X}{d t} \\
\frac{d Y}{d t} \\
\frac{d \Psi}{d t}
\end{array}\right]=\frac{r}{2}\left[\begin{array}{cc}
\cos (\Psi) & \cos (\Psi) \\
\sin (\Psi) & \sin (\Psi) \\
\frac{1}{l} & -\frac{1}{l}
\end{array}\right] \cdot\left[\begin{array}{l}
\Omega_{r} \\
\Omega_{l}
\end{array}\right]
$$

where :

- $\quad \mathrm{X}$ and $\mathrm{Y}$ are the coordinate of the robot in the environment frame,

- $\Psi$ is the orientation in the same frame,

- $\Omega_{1}$ and $\Omega_{\mathrm{r}}$ are the angular speed of the left and right wheels,

- $\quad \mathrm{r}$ is the wheel's rayon,

- 1 is the distance between two wheels.

\subsection{The Neurocontroller}

As explain in (Floreano, 1994) and (Haussler, 1995) genetic algorithms can be used to train and optimize artificial neural networks (ANNs). Such solution has been selected here for its interesting link with biology and its anteriority in the field of robotics. Previous works (Braitenberg, 1986) prove that simple neurocontroller can be used to performed obstacle avoidance. Assuming that the grasping task is similar to an obstacle avoidance behavior from the neurocontroller point of view, the same structure for the neural network without hidden layer has been chosen (Figure 4 shows the neural network).

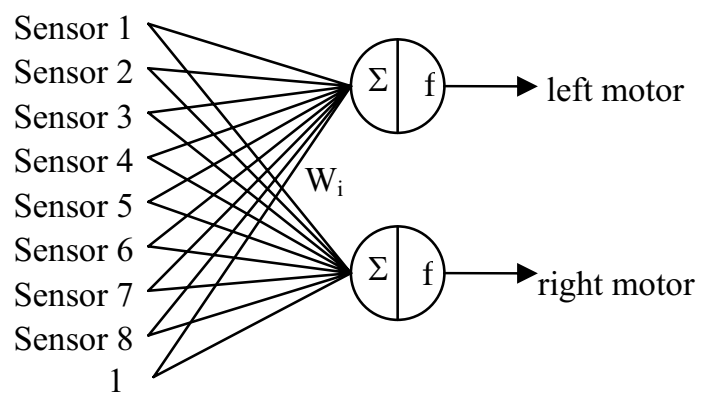

Figure 4: Neurocontroller's structure.

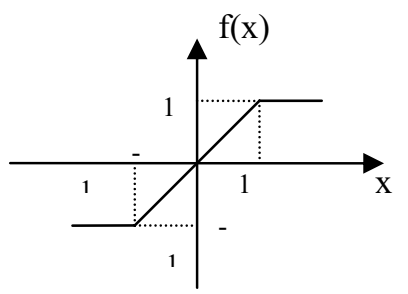

Figure 5: Transfer function for each perceptron.

In order to homogenize the simulation and the result analysis, all the data are scaled in the interval $[-1 ; 1]$. For example, in the case of the wheel's angular speed: +1 applied to the motors is equivalent to the maximum speed $\left(0.1 \mathrm{rad} . \mathrm{s}^{-1}\right)$. For the same reason, the synaptic weights are bounded in the same interval and the output of the perceptron is also bounded (Figure 5) to reproduce the mechanical characteristics of the motors. Proximity sensor's data is applied on the input of the network that computes the command on each motor. Note that a synaptic link with a constant value applied to the input (equal to one) has been added allowing the robot to move when none of the sensors are providing a value, i.e. when all the $c_{i}$ are equal to zero. As usual, the neural network is just a friendly representation of a mathematical expression (equation 2). In this 
equation, all the parameters are known except the 18 synaptic weights, optimized by the evolutionary algorithm.

$$
\begin{gathered}
\Omega_{l}=f\left(\sum_{i=1}^{8} w_{i} \cdot c_{i}+w_{9}\right) \\
\Omega_{r}=f\left(\sum_{i=1}^{8} w_{i+9} \cdot c_{i}+w_{18}\right)
\end{gathered}
$$

where :

- $\Omega_{1}$ and $\Omega_{\mathrm{r}}$ : command applied on the motors,

- $\mathrm{w}_{\mathrm{i}}, \mathrm{i} \in[1 ; 9]$ : synaptic weight for the left motor,

- $\mathrm{w}_{\mathrm{i}}, \mathrm{i} \in[9 ; 18]$ : synaptic weight for the right motor,

- $\quad c_{i}$ : distance detected by the sensor $\mathrm{i}$.

\subsection{The Evolutionary Algorithm}

The algorithm is based on a classical genetic approach described as follow.

\subsubsection{Chromosome}

As the structure of the neurocontroller has been fixed, only the synaptic weights have to be optimized. The chromosomes are only containing these weights. An intuitive approach consists in coding the weights in binary, providing a series of 0 and 1. The drawback of this approach results in the most important influence of most significant bit during crossovers and mutations. To avoid this problem, an elementary component of the chromosome is not a 0 or a 1 , but the weight himself as described in table 1 .

Table 1: Structure of a chromosome.

\begin{tabular}{|l|l|l|l|l|l|l|}
\hline $\mathrm{W}_{1}$ & $\mathrm{~W}_{2}$ & $\ldots$ & $\mathrm{w}_{\mathrm{i}}$ & $\ldots$ & $\mathrm{w}_{17}$ & $\mathrm{w}_{18}$ \\
\hline
\end{tabular}

\subsubsection{Crossovers}

Crossovers are performed with two individuals selected from previous generation. The selection of the individual is based on the roulette-wheel reproduction as described in (Nolfi, 2000) that allows the best individual to be statistically selected more frequently. The probability for an individual $n$ to be selected is given by the equation 3 .

Once the two parents are selected, one of them is randomly selected (each with a probability of 0.5 ) to provide the first gene, and this process is repeated 18 times (one for each weight). The crossover's strategy is multipoint as described in (Mitchell, 1997).

$$
P_{n}=\frac{f_{n}}{\sum_{i=0}^{N} f i}
$$

where :

- $P_{n}$ : probability of selection for the individual $n$,

- $\mathrm{f}_{\mathrm{j}}$ : fitness of the individual $j$,

- $\mathrm{N}$ : number of individuals in the previous generation

\subsubsection{Mutation}

According to the strategy chosen, the mutation process is very important. Assume that the optimal weights for a given gene aren't present in none of the 100 individuals: without mutation, it is strictly impossible to find the optimal solution. Mutations are indeed very important and the mutation rate must be high enough to ensure a good exploration of the space. The mutation process is performed after the crossovers; $10 \%$ of the new individuals are randomly selected. For each individual a gene is randomly selected and replaced by a random value in the range $[-1,1]$. For each draw, the probability is uniform.

\subsubsection{Fitness Function}

As our goal is a grasping task, the fitness function must be linked with the explored area. The environment is sampled with a sampling rate of 30 $\mathrm{cm}$ for each axis. As the size of the environment is $3 \mathrm{~m} \times 3 \mathrm{~m}$ the space is divided into 100 squares. At the end of the evaluation of each individual, the fitness function is computed with the equation 4. Figure 6 shows a snapshoot of the simulator.

$$
f_{j}=\frac{N_{\text {explored }}}{N_{\text {Total }}}
$$

where :

- $f_{\mathrm{j}}$ : fitness of the individual $\mathrm{j}$,

- $\quad N_{\text {explored }}:$ number of squares explored

- $N_{\text {Total }}$ : total number of squares

\subsubsection{Parameters}

In the first version of the simulator, a noise was added on the motors. After analyzing the results we discovered that this noise prevented from comparing 
the results. We decided to eliminate this noise in order to make the simulator deterministic. For the same reason, the initial position of the robot is always located at the same place (at the top left, c.f. Figure 6) to prevent from favoring individuals.

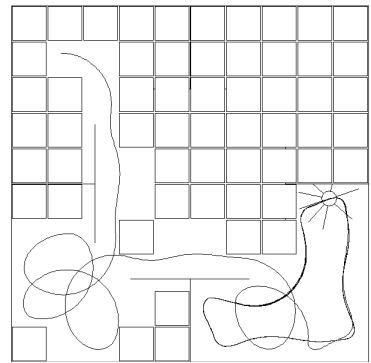

Figure 6: Snapshoot of the simulator showing the trajectory of the robot and the explored area. The current fitness of the robot is 0.43 (43 explored squares divided by 100 total squares).

Note that collisions between robot and walls are considered. As the neurocontroller has been designed without hidden layer, it is impossible for a jammed robot to escape from a collision. To decrease the computation time, when a robot is jammed its evaluation is stopped and the fitness is computed. Table 2 describes the parameters of the simulator.

Table 2: Parameters of the simulations.

\begin{tabular}{|l|c|c|}
\hline \multicolumn{1}{|c|}{ Description } & Name & Value \\
\hline Wheel's rayon & $\mathrm{r}$ & $0.05 \mathrm{~m}$ \\
\hline Distance between wheels & 1 & $0.1 \mathrm{~m}$ \\
\hline Sensor's range & $\mathrm{Sr}$ & $0.2 \mathrm{~m}$ \\
\hline $\begin{array}{l}\text { Maximum angular speed of } \\
\text { the wheels }\end{array}$ & $\Omega_{\max }$ & $0.1 \mathrm{rad} . \mathrm{s}^{-1}$ \\
\hline Size of the environment & - & $3 \times 3 \mathrm{~m}$ \\
\hline Sampling rate (space) & - & $0.3 \mathrm{~m}$ \\
\hline Size of the population & $\mathrm{N}_{\text {individual }}$ & $100 \mathrm{ind}$. \\
\hline Mutation rate & - & $10 \%$ \\
\hline Sampling rate (time) & $\Delta_{\mathrm{t}}$ & $0.01 \mathrm{~s}$ \\
\hline Duration of an evaluation & - & $30 \mathrm{~s}$ \\
\hline Number of generations & - & $250 \mathrm{gen}$. \\
\hline
\end{tabular}

\section{STANDARD BEHAVIORS}

\subsection{Standard Experiments}

For each environment a neurocontroller has been trained. This controller is considered as the standard behavior in the following. The evolution of the 250 generations lasts around two hours on a desktop computer. Figure 7 shows the evolution of an individual in the environment c. Figure 8 shows the evolution of the fitness. For each environment, an efficient strategy has been generated that confirms the relevance of the used parameters.

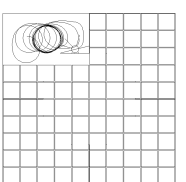

a.

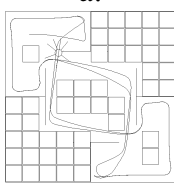

d.

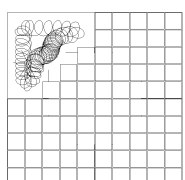

b.

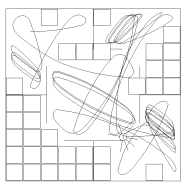

e.

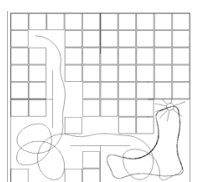

c.

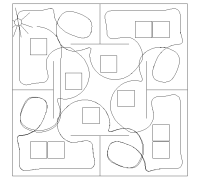

f.
Figure 7: Evolution of the robot's behavior in the environment c. (see Fig.1) at generations 2,3,6,13,17 and 162 with respective fitness: $0.15,0.19,0.43,0.51,0.66$ and 0.93 .

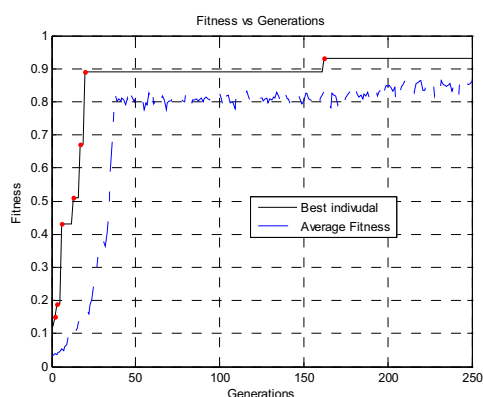

Figure 8: The fitness of the individuals (Fitness of the best individual (continuous line) for each generation and average (dotted line) of the whole population).

Analyzing the results shows that efficient behaviors can be classified in three categories:

- performing epicycloidals trajectories (Figure 9.a),

- moving straight and avoiding obstacles (Figure 9.b)

- wall following (Figure 9.c)

Best strategies are usually a mixed of the previous behaviors (Figure 9.d)

\subsection{Swapping the Environments}

In order to evaluate how generic are the produced behaviors, each individual is placed in the seven other environments. Note that the genetic process is stopped. Table 3 summarizes the results. For example bolded $38 \%$ presents the performance in the environment $\mathrm{a}$ of the individual trained in the 
environment d. Grey cells show the best performance for each environment. This agrees with the diagonal that represent the performance of each individual in "its" environment except for environment $\mathrm{d}$. and e. where individuals are equally ranked, probably due to the fact that environments $\mathrm{d}$. and e. are quiet similar.
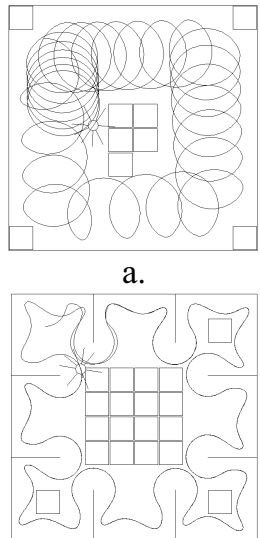

c.

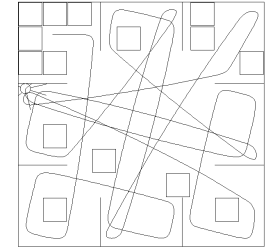

b.

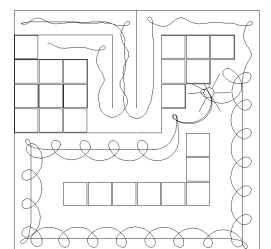

d.
Figure 9: Examples of strategies used for exploring the environment.

This preliminary result allows us two conclusions: the neurocontroller produced by the genetic process cannot be considered as generic. An nice illustration is shown on table 3.: the performance in the environment $\mathrm{g}$. is very poor except for the individuals trained in this kind of environment. These results also confirm that genetic algorithm may be considered as generic: the produced behavior is nicely adapted to the trained environment, on table 3 . the best fitness are always located on the diagonal. The last part of the paper will discuss about the best strategy for generating generic neurocontrollers.

\section{GENERIC NEUROCONTROLLERS}

\subsection{Random Selection of the Environment}

The first idea for building generic neurocontrollers consists in mixing the environments during the evolution. A new simulation has been performed, but the environment is now randomly selected for each generation. After 1500 generations, the synaptic weights never converge to a stable value. Figure 10 shows the evolution of the fitness during the 200 first generations (no changes were observed after). Compared to Figure 8 this evolution cannot be considered as satisfying. Results have shown that random selection of the environment makes the global system non-deterministic and prevents the genetic algorithm from finding the optimal solution. To avoid this problem, each individual is now trained in the eight environments.

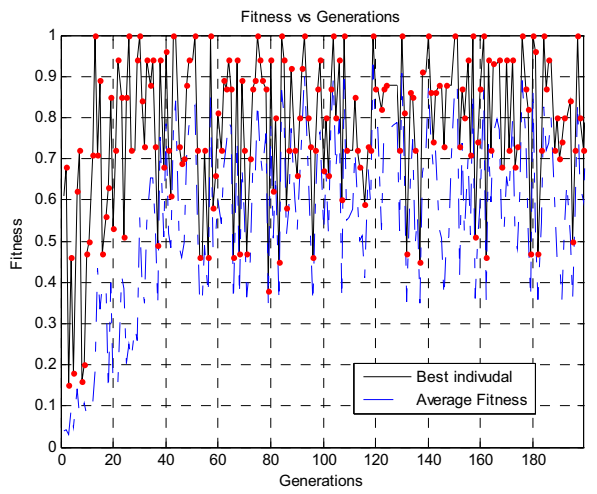

Figure 10: Fitness versus generations.

Table 3: Performance of the best individual of the final generation in the seven other environments.

Explored environment

\begin{tabular}{|c|c|c|c|c|c|c|c|c|}
\hline \multirow{9}{*}{ 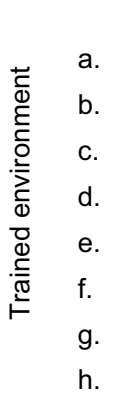 } & a. & b. & c. & d. & e. & f. & g. & h. \\
\hline & $96 \%$ & $70 \%$ & $6 \%$ & $9 \%$ & $12 \%$ & $10 \%$ & $1 \%$ & $1 \%$ \\
\hline & $94 \%$ & $98 \%$ & $4 \%$ & $11 \%$ & $14 \%$ & $11 \%$ & $2 \%$ & $2 \%$ \\
\hline & $14 \%$ & $13 \%$ & $93 \%$ & $92 \%$ & $33 \%$ & $20 \%$ & $15 \%$ & $15 \%$ \\
\hline & $38 \%$ & $62 \%$ & $9 \%$ & $100 \%$ & $86 \%$ & $81 \%$ & $11 \%$ & $11 \%$ \\
\hline & $7 \%$ & $7 \%$ & $7 \%$ & $100 \%$ & $86 \%$ & $5 \%$ & $4 \%$ & $4 \%$ \\
\hline & $41 \%$ & $65 \%$ & $8 \%$ & $9 \%$ & $9 \%$ & $85 \%$ & $14 \%$ & $14 \%$ \\
\hline & $38 \%$ & $62 \%$ & $17 \%$ & $23 \%$ & $84 \%$ & $77 \%$ & $77 \%$ & $72 \%$ \\
\hline & $44 \%$ & $64 \%$ & $90 \%$ & $93 \%$ & $84 \%$ & $83 \%$ & $61 \%$ & $76 \%$ \\
\hline
\end{tabular}




\subsection{Evolution in the Eight Environments}

For the reason explained in the previous section, each individual is now successively trained in the eight environments. The fitness is similar to the previously described equation 4 (equivalent to the average performance in the eight environments). The experiment lasts about fifteen hours (eight times longer than for one environment). The evolution of the fitness is now asymptotic (similar to figure 8) that proves the convergence of the genetic algorithm. Examples of trajectories are shown on figure 11 .

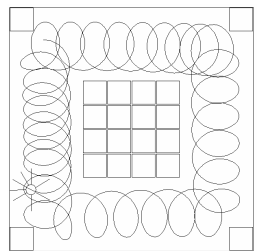

a.

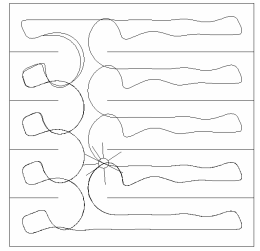

c.

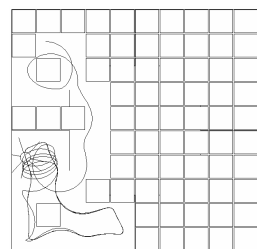

b.

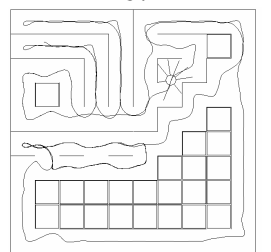

d.
Figure 11: Example of trajectories in four environments.

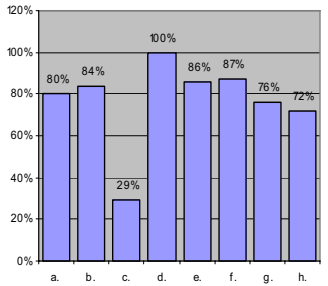

a.

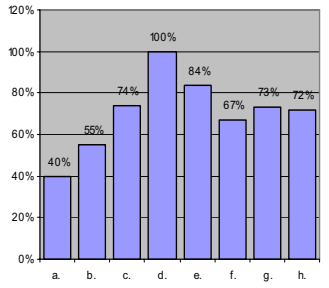

b.
Figure 12: Performance of the best individual (last generation) in the eight environments.

Figure 12.a. shows the fitness in each environment. The global performance is satisfying $(76.75 \%)$ to the detriment of the environment c. (only 29\%). Figure 11.b. shows the trajectory of the robot in this environment; the robot is quickly jammed in a dead end. However it's hard to conclude about the adaptability of the neurocontroller. Indeed, it is clear that the performance in the environment $\mathrm{c}$. has been sacrificed in favour of global fitness. Considering that generic means able to perform a high fitness in any situations, this goal isn't reach.

\subsection{Increasing the Last}

To avoid having a "sacrificed" environment, we performed the previous experiment with a new fitness function. The average isn't longer considered. Each individual is evaluated in the eight environments and the new fitness is the performance in the worst environment. For example, on figure 12.a, the performance used to compute crossovers is the weakest: $29 \%$ (environment c.).

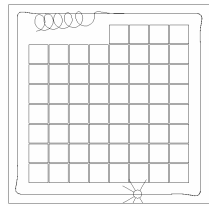

a.

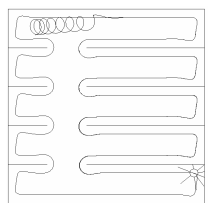

d.

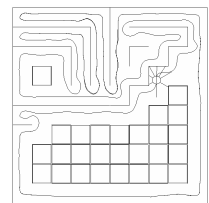

g.

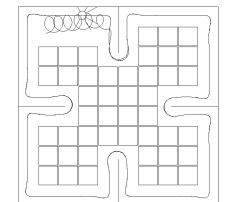

b.

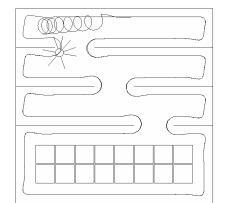

e.

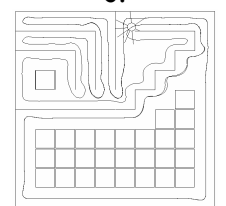

h.

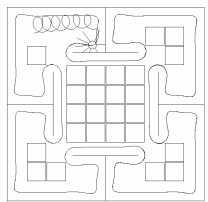

c.

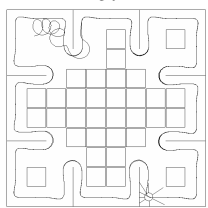

f.
Figure 13: Trajectory of the robot in each environment.

Figure 12.b shows the performance in each environment of the best individual (last generation). The worst performance is $40 \%$ in the environment a. (slightly better than the previous 29\%). The trajectories of the robot are clearly based on a wall following strategy visible on figure 13. As the environment a. has no wall (except the outline walls) the performance is poor. In spite of this, this result is encouraging. The average performance is $70.62 \%$, not so far from the previous $76.75 \%$. This means that the global performance isn't too much affected. Even if these results are globally worst, it stays encouraging. Probably that the chosen neurocontroller (without hidden layer) does not allow the robot to perform a high performance in the eight environments at the same time. These results tend to show that taking the performance of the worst case may provide more generic controllers than averaging the fitness 


\section{CONCLUSIONS}

We presented in this paper some experiments based on the grasping task (for example an autonomous vacuum robot). These experiments are based on the genetic evolution of a neurocontroller without hidden layer. In the first part we evolved in simulation eight neurocontrollers (each in a given environment). The neurocontroller were swapped and the performances in the other environments were evaluated. For some researchers, there is sometime a mix-up between the genetic algorithm and the generated behavior. We've shown that genetics algorithms can be easily adapted with the same parameters to several problems. We've also shown that the generated neurocontroller is dedicated to the trained environment. It means that genetics algorithms are generic, contrary to neurocontrollers that are dedicated. This result can probably be extended to all the parameters of the evaluation: noise, robot's hardware, battery charge, etc.

In the second part of the paper, several strategies were experimented to produce generic neurocontrollers. First, the evaluation of the individual was done in the eight environments and the average performance was used for the fitness computation. This experiment provides good results except in one environment where the fitness was very poor. In the final experiment the performance in the worst environment was used to compute the fitness. The global performance is slightly smaller than in the previous experiment, but the performance is more distributed in the environments. Generating generic controllers using genetic algorithms stay a complex problem but we've shown that taking the worst case for evaluating the individual may be a first step in the automatic generation of generic neurocontrollers.

\section{REFERENCES}

Jansen T. and Wegener I., 2002, On the analysis of evolutionary algorithms: A proof that crossover really can help. Algorithmica, Springer New York, Volume 34, Number 1 / July, 47-46.

Bäck T., 1996, Evolutionary algorithms in theory and practice: evolution strategies, evolutionary programming, genetic algorithms. Oxford University Press.

Floreano D. and Mondada F., 1994, Automatic Creation of an Autonomous Agent: Genetic Evolution of a Neural Network Driven Robot. 3rd International Conference on Simulation of Adaptive Behavior (SAB'94).
Mondada F., Franzi E. and Ienne, P., 1993, Mobile Robot Miniaturization: A Tool for Investigation in Control Algorithms, 3rd International Symposium on Experimental Robotics III, October 28-30, 501-513.

Floreano D. and Mondada F., 1998, Evolutionary Neurocontrollers for Autonomous Mobile Robots. Neural Networks, 11(7-8), 1461-1478.

Nolfi S. and Floreano D., 2000, Evolutionary Robotics: The Biology, Intelligence, and Technology of Selforganizing Machines. Bradford book, MIT Press, Cambridge, Massachusetts.

Ahuactzin J.M., Talbi E-G, Bessiere P. and Mazer E.,1992 , Using genetic algorithms for robot motion planning. European Conference on Artificial Intelligence (ECAI92), Wien (Austria).Yamasaki F., Endo K., Kitano H. and Asada M., 2002, Acquisition of humanoid walking motion using genetic algorithm Considering characteristics of servo modules. Robotics and Automation, Proceedings. ICRA ' 02. IEEE International Conference on Robotics and Automation, 3123-3128.

Arkin R.C., 1992, Cooperation without Communication, Multiagent Schema-Based Robot Navigation, Journal of Robotic Systems, Vol. 9 (3), avril , 351-364.

Lucidarme P. and Simonin O.,2006, Le robot mobile Type 1, Journée des démonstrateurs en automatique, Angers, France.

Haussler A., Li Y., Ng K.C., Murray-Smith D.-J. and Sharman, K.C., 1995, Neurocontrollers designed by a genetic algorithm. First conference on genetic algorithms in engineering systems: innovations and applications, Sheffield, UK, Publ. No. 414.

Braitenberg V., 1986, Vehicules - experiments in synthetic psychology, Bradford books.

Mitchell T.M., 1997, Machine learning. McGraw-Hill Science. 